\title{
Detección serológica de Chlamydophila psittaci en psitácidos en cautiverio de la Región del Biobío, Chile
}

\author{
SEROLOGICAL DETECTION OF CHLAMYDOPHILA PSITTACI IN PSITTACIDS IN CAPTIVITY \\ of THE Biobío Region, ChILE \\ Karla Pinto $^{1}$, Felipe Villalobos ${ }^{2}$, Christof Fischer ${ }^{1}$, Carlos Barrientos ${ }^{1}$, \\ Daniel González-Acuña ${ }^{3}$, Ignacio Troncoso ${ }^{4,5}$
}

\section{Resumen}

Los loros (Aves: Psitacidae), además de ser una de las mascotas de mayor incremento en Chile en los últimos años, constituyen importantes reservorios de patógenos, incluída la bacteria zoonótica Chlamydophila psittaci. El objetivo del estudio fue determinar la presencia de anticuerpos séricos contra C. psittaci en 30 loros provenientes de tres criaderos privados de la región del Biobío, mediante la detección de anticuerpos tipo IgG a través un ELSA comercial (ELISA InmunoComb $\left.{ }^{\circledR}\right)$. Tres loros adultos (3/24) resultaron seropositivos (10\% de prevalencia) y ningún juvenil (0/6). Los psitacidos positivos corespondieron a una cachaña (Enicognathus ferrugineus), un choroy (Enicognathus leptorhynchus) y una amazona (Amazonas pretrei), todos provenientes del mismo criadero. La cachaña y el choroy son especies nativas de Chile.

Palabras clave: Psitaciformes; clamidiosis, ELISA; anticuerpos; serología

\footnotetext{
${ }^{1}$ Escuela de Medicina Veterinaria, Facultad de Recursos Naturales y Medicina Veterinaria, Universidad Santo Tomás, Concepción, Chile

${ }^{2}$ Escuela de Medicina Veterinaria, Facultad de Recursos Naturales y Medicina Veterinaria, Talca, Chile

${ }^{3}$ Facultad de Ciencias Veterinarias, Universidad de Concepción, Chillán, Chile

${ }^{4}$ Escuela de Medicina Veterinaria, Facultad de Recursos Naturales y Medicina Veterinaria, Universidad Santo Tomás, Temuco, Chile

${ }^{5}$ E-mail: ignaciotroncoso@santotomas.cl
}

Financiamiento: Facultad de Recursos Naturales y Medicina Veterinaria, Universidad Santo Tomás, 
The parrots (Birds: Psitacidae), besides being one of the pets of greater increase in Chile in recent years, are important reservoirs of pathogens, including the zoonotic bacterium Chlamydophila psittaci. The objective of the study was to detect the presence of serum antibodies against $C$. psittaci in 30 parrots from three private breeding sites in the Biobío Region, through the detection of IgG antibodies by a commercial ELSA kit (ELISA InmunoComb $\left.{ }^{\circledR}\right)$. Three adult parrots $(3 / 24)$ were seropositive $(10 \%$ prevalence) and none of the juveniles $(0 / 6)$. The positive psitacids corresponded to an austral parakeet (Enicognathus ferrugineus), a slender-billed parakeet (Enicognathus leptorhynchus) and an Amazon parrot (Amazonas pretrei), all from the same hatchery. The first two species are native species of Chile.

Key words: Psittaciformes; chlamydiosis, ELISA; antibodies; serology

\section{INTRODUCCIÓN}

Según Abarca et al. (2002), la tenencia de mascotas exóticas en Chile ha ido creciendo en los últimos años, siendo los loros uno de los grupos de mayor interés. Reyes (2011) realizó una encuesta a 496 hogares, 13 clínicas veterinarias y cinco tiendas de mascotas de la ciudad de Talca determinando que los Psitaciformes fue el grupo más abundante $(70 \%, 89 / 127)$ en la tenencia de mascotas exóticas, especialmente en dos de ellas: catita australiana Melopsittacus undulatus (88) y aves del género Agapornis.

De acuerdo con la información registrada, las aves psitáìcidas en cautiverio en Chile corresponden a 2913 loros exóticos (excluyendo a catitas y cotorritas ninfas [Nymphicus hollandicus]) y 340 corresponden a loros nativos en cautiverio (choroy [Enicognathus leptorhynchus], cachaña [Enicognathus ferrugineus] y tricahue [Cyanoliseus patagonus bloxami]). Estos uiltimos son encontrados mayoritariamente en zoológicos, criaderos, centros de exhibición y de rehabilitación de fauna silvestre (DEPROREN, 2003).
Las aves cautivas pueden actuar como reservorios de enfermedades zoonóticas, hecho que se agrava por el estrecho contacto con el ser humano (Dabanch, 2003). Aunque se reconoce que la bacteria Chlamydophila psittaci es capaz de afectar aves de más de 30 órdenes, incluyendo especies migratorias de aves acuáticas, gaviotas, aves costeras, palomas, paseriformes, etc. (Kaleta y Taday, 2003), las aves del orden Psitaciformes corresponden a uno de los principales reservorios de esta bacteria, microorganismo responsable de la clamidiosis o psitacosis (Martínez y Tinetti, 2007). Se ha determinado, además, que algunas cepas de Clamidia, que normalmente no son patógenas para aves silvestres, pueden ser altamente virulentas para las aves domésticas y seres humanos (Hubalek, 2004).

En Chile se han publicado solo tres estudios relacionados con esta bacteria. Un estudio de seroprevalencia de C. psittaci en aves psitacidas de la zona central (González, 2006), trabajo en el cual se obtuvo un $22.4 \%$ de prevalencia y dos trabajos realizados en palomas silvestres (Columba livia), uno realizado por González-Acuña et al. (2007) en la ciudad de Chillán, y el segundo por 
Altamirano (2007) en la ciudad de Valdivia, quienes obtuvieron 11 y $16.9 \%$ de seroprevalencia con las técnicas de ELISA y fijación de complemento, respectivamente.

Bajo este contexto, el presente estudio tuvo como objetivo detectar a través de la técnica serológica de ELISA Inmunocomb ${ }^{\circledR}$ la presencia de anticuerpos contra $C$. psittaci en psitácidos en cautiverio provenientes de tres criaderos particulares de la región del Biobío.

\section{Materiales y Métodos}

\section{Lugar y Aves de Estudio}

El presente estudio se realizó en ejemplares de psitácidos provenientes de tres criaderos particulares de la Región del Biobío, Chile. Se efectuó un muestreo dirigido durante enero de 2014 a 30 ejemplares ( 24 adultos y 6 juveniles) de loros, criados en jaulas comunes que incluían ocho especies: 12 periquitos australianos (Melopsittacus undulatus), un inseparable de Fischer (Agapornis fischeri), un kakariqui de frente roja (Cyanoramphus novaezelandiae), dos ninfas (Nymphicus hollandicus), siete rosellas comunes (Platycercus eximius), cuatro cachañas (Enicognathus ferrugineus), dos choroyes (Enicognathus leptorhynchus) y una amazona-roja de anteojos (Amazona pretrei).

\section{Muestras y Análisis}

Se obtuvo $1 \mathrm{ml}$ de sangre de cada ave mediante venopunción en la vena braquialulnar. La sangre fue depositada sobre un disco de papel (paper disk) especial para la colecta de sangre y posterior análisis. Los discos se depositaron en un tubo vacutainer estéril sin anticoagulante debidamente rotulado, y transportados en una caja conservadora a $4{ }^{\circ} \mathrm{C}$ a las instalaciones de la Universidad Santo Tomás, sede Concepción.
Como método diagnóstico se utilizó el kit comercial Immunocomb ${ }^{\circledR}$ (Biogal, Kibbutz Galed, Israel), que corresponde a una prueba diagnóstica de ELISA indirecta (Beeckman y Vanrompay, 2009), diseñada para determinar títulos de inmunoglobulina $\mathrm{G}(\mathrm{IgG})$ de diferentes serovares patógenos de C. psittaci con un alto índice de sensibilidad (95\%) y especificidad (85\%), según Bendheim et al. (1996). La prueba se realizó siguiendo las indicaciones del fabricante.

\section{Análisis Estadístico}

El estudio fue de tipo descriptivo y transversal. Para la evaluación de los resultados del análisis serológico se formaron dos grupos de aves según los rangos de edad: juvenil (5 ejemplares) y adulto ( 25 ejemplares) (Forshaw, 2006), según especie y por criadero (A, B, C). Se determinó la seropositividad mediante el cálculo porcentual simple, mientras que para determinar diferencias estadísticas entre criaderos y para la variable edad se aplicó el test de Fisher utilizando el software Epi 6.

\section{Resultados y Discusión}

Se obtuvo reacción positiva a C. psittaci en tres de las 30 muestras analizadas $(10 \%$ de prevalencia). Los tres ejemplares positivos fueron adultos $(12.5 \%)$, provenientes del mismo criadero, siendo estos E. ferrugineus, E. leptorhynchus y A. pretrei (Cuadro 1).

El presente estudio es el primero en encontrar serología positiva a $C$. psittaci en dos especies nativas de Chile (cachaña, choroy) (Olson, 1995; Jaramillo, 2003); sin embargo, el resultado serológico fue inferior al obtenido por González (2006) en muestras de sangre de 10 poblaciones de aves Psittaciformes en cautiverio localizadas en la Región Metropolitana de Chile, encontrando anticuerpos séricos contra $C$. psittaci en 11 de 49 aves (22.4\%), utilizando un test de 
Cuadro 1. Seroprevalencia de Chlamydia psittaci por especie de psitacidos

\begin{tabular}{lccc}
\hline Especie & $\begin{array}{c}\text { Aves } \\
\text { muestreadas } \\
\text { (n) }\end{array}$ & $\begin{array}{c}\text { Grupo } \\
\text { etario }\end{array}$ & $\begin{array}{c}\text { Positivas a } \\
\text { C. psittaci } \\
\text { (n) }\end{array}$ \\
\hline Periquito australiano (Melopsittacus undulatus) & 7 & Adulto & 0 \\
Inseparables (Agapornis fischeri) & 5 & Juvenil & 0 \\
Kakariki (Cyronamphus novaezelandiae) & 1 & Adulto & 0 \\
Ninfa (Nymphicus hollandicus) & 1 & Adulto & 0 \\
& 1 & Adulto & 0 \\
Rosella (Platycercus eximius eximius) & 1 & Juvenil & 0 \\
Cachaña (Enicognathus ferrugineus) & 7 & Adulto & 0 \\
Choroy (Enicognathus leptorhynchus) & 4 & Adulto & 1 \\
Amazonas (Amazona pretrei) & 2 & Adulto & 1 \\
\hline Total & 1 & Adulto & 1 \\
\hline
\end{tabular}

ELISA considerado como efectivo método de «screening» para detectar anticuerpos antiC. psittaci (Bendheim et al., 1996); técnica que ha sido empleada por diversos autores en aves psitácidas (Ryll et al., 1994; Reither, 1999; Raso et al., 2002). Según Magnino et al. (2009) y Moráis et al. (2013), el kit comercial Immunocomb ${ }^{\circledR}$ es actualmente la prueba de ELISA disponible más adecuada por su alta sensibilidad y especificidad.

Otros estudios en Chile, como el de González-Acuña et al. (2007) analizando 100 palomas en Chillán con el kit comercial de ELISA (IDEXX), test con sensibilidad y especificidad de $100 \%$ (Talazadeh et al., 2014) encontraron 11 individuos positivos ( $11 \% \mathrm{de}$ prevalencia); mientras que Altamirano (2007), en Valdivia, encontró 10 de 59 aves positivas ( $16.9 \%$ de prevalencia) usando la prueba de fijación del complemento ( $44.4 \%$ de sensibilidad y 99.6\% de especificidad) (Pianciola $e t$ al., 2010). Es importante considerar que la infección en Columbiformes se perpetua de manera primaria mediante la transmisión de la bacteria desde los adultos a los juveniles (Altamirano, 2007).
Tomando en consideración investigaciones internacionales, Herrmann et al. (2006) obtuvo $10 \%$ de prevalencia para $C$. psittaci en gaviotas en las Islas Faroe utilizando la técnica de PCR, mientras que Blomqvist et al. (2012) demostraron 1.3\% de prevalencia en $C$. psittaci en 319 halcones peregrinos (Falco peregrinus) y águilas de cola blanca (Haliaeetus albicilla) en Suecia con técnicas moleculares.

Los resultados del presente estudio difieren de los reportados por Dovc et al. (2007), quienes registraron presencia de antígenos clamidiales en $15.2 \%$ de 46 periquitos australianos (Melopsittacus undulatus), pero todos los sueros resultaron seronegativos. Situación opuesta demostraron Raso et al. (2002), quienes obtuvieron $37 \%$ de seroprevalencia en guacamayos silvestres (Amazona aestiva, A. amazónica, A. farinosa, A. ochrocephala, A. pretrei, A. rhodocorytha, $A$. vinacea y $A$. Xanthops) en Brasil a través de la técnica de inmunofluorescencia directa. Así mismo, Schettler et al. (2003) detectaron 74\% de muestras positivas en aves de rapiña de vida 
libre en Alemania mediante PCR; PruknerRadovcic et al. (2005) encontraron el 95.6\% de muestras positivas a C. psittaci en 182 palomas en Zagreb, Croacia; y Monsalve et al. (2011) obtuvieron $85 \%$ de seropre-valencia en Colombia mediante ELISA indirecta en loros del género Amazona en cautiverio.

La baja seroprevalencia obtenida en este estudio puede estar relacionado a que gran parte de las aves muestreadas (76.6\%) pertenecen a especies del «Viejo Mundo», aves que poseen una menor susceptibilidad a la infección por C. psittaci (Gerlach, 1994). Además, las aves se encontraban libres de factores estresantes, ya que estaban sometidas a un manejo en cautividad sin sobrepoblación, con alimentación balancea$\mathrm{da}$, en condiciones ambientales apropiadas, con poca movilidad de aves portadoras $\mathrm{y}$, generalmente, en ausencia de otras enfermedades concomitantes.

Con relación a la variable edad, González-Acuña et al. (2007) encontraron valores de positividad para $C$. psittaci similares para juveniles y adultos, a diferencia del presente estudio donde todos los casos positivos fueron en aves adultas. Esto contrasta con el hecho que las aves jóvenes son más susceptibles a esta enfermedad que las aves adultas (Sachse et al., 2009), situación que podría explicarse por el reducido número de aves seropositivas encontrado. Así mismo, Altamirano (2007), reportó una menor frecuencia de muestras seropositivas en palomas juveniles que en adultas.

Es necesario recalcar que un ave seropositiva puede ser un potencial portador de clamidiosis (Ruppanner et al., 1984), una de las principales enfermedades zoonóticas de aves (Ramsay, 2003), de alta relevancia en salud pública y que sumada a la popularidad de las aves psitácidas como mascotas, aumenta el riesgo de contagio en la población humana, de ahí la importancia de realizar posteriores estudios de aislamiento y caracterización genético molecular de las cepas aviares y humanas de C. psittaci que circulan en Chile.

\section{Conclusiones}

El presente estudio pionero detectó serología positiva para Chlamydophila psittaci en dos especies nativas de psitacidos de Chile (cachaña [Enicognathus ferrugineus] y choroy [Enicognathus leptorhynchus]) y en Amazona pretrei.

\section{Literatura Citada}

1. Abarca K, Dabanch J, Jofrè L, Olivares R, Perret C, Rodríguez J. 2002. Tenencia de mascotas en población sana e inmunodeprimida de Santiago. En: XIX Congreso Chileno de Infectología. Santiago, Chile.

2. Altamirano DA. 2007. Determinación de anticuerpos contra Chlamydophila psittaci y pesquisa de Salmonella sp. en palomas (Columba livia Gmelin, 1789) de la ciudad de Valdivia. Tesis de Médico Veterinario.Valdivia, Chile: Univ. Austral de Chile. 35 p.

3. Beeckman DS, Vanrompay DC. 2009. Zoonotic Chlamydophila psittaci infections from a clinical perspective. Clin Microbiol Infec 15: 11-17. doi: 10.1111/j.1469-0691.2008.02669.x

4. Bendheim U, Wodovski I, Ordonez M, Naveh A. 1996. Development of an ELISA kit for antibody determination in birds including poultry and psittacines. Isr J Vet Med 51: 147-148.

5. Blomquist $M$, Christerson L, Waldenström J, Lindberg P, Helander B, Gunnarsson G, et al. 2012. Chlamydia psittaci in birds of prey, Sweden. Infect Ecol Epidemiol 2. doi: https://doi.org/10.3402/iee.v2i0.8435

6. Dabanch J. 2003. Zoonosis. Rev Chil Infect 20: 47-51. doi: 10.4067/S071610182003020100008

7. [DEPROREN] Departamento de Proteccioin de los Recursos Naturales Renovables, Servicio Agrícola y Ganadero. 2003. Vida Silvestre. 
8. Dovc A, Slavec B, Lindtner-Knific R, Zorman-Rojs O, Racnik J, Golja J, Vlahovic K. 2007. Study of Chlamydophila psittaci outbreak in budgerigars. B Vet I Pulawy 51: 343-346.

9. Forshaw MJ. 2006. Parrots of the world: an identification guide. USA: Princeton University Press. 336 p.

10. Gerlach H. 1994. Clamydia. In: Avian medicine: principles and applications. USA: Abridged. p 984-996.

11. González G 2006. Estudio serológico de Chlamydophila psittaci, Salmonella spp, virus pox aviar, adenovirus y virus polioma en aves del orden psitaciforme en cautiverio en Chile Central. Tesis de Médico Veterinario. Santiago de Chile: Univ. de Chile, Santiago, Chile. 110 p.

12. González-Acuña D, Silva F, Moreno $L$, Cerda F, Donoso S, Cabello J, López J. 2007. Detección de algunos agentes zoonóticos en la paloma doméstica (Columba livia) en la ciudad de Chillán, Chile. Rev Chil Infectol 24: 199203. doi: 10.4067/S0716-10182007000300004

13. Herrmann B, Persson H, Jensen JK, Joensen HD, Klint M, Olsen B. 2006. Chlamydophila psittaci in fulmars, the Faroe Islands. Emerg Infect Dis 12: 330332. doi: 10.3201/eid1202.050404

14. Hubálek Z. 2004. An annotated checklist of pathogenic microorganisms associated with migratory birds. J Wildlife Dis 40: 639-659. doi: 10.7589/ 0090-3558-40.4.639

15. Jaramillo A. 2003. Birds of Chile. USA: Princeton Field Guides. 142 p.

16. Kaleta EF, Taday EM. 2003. Avian host range of Chlamydophila spp based on isolation, antigen detection and serology. Avian Pathol 32: 435-462. doi: 10.1080/03079450310001593613

17. Magnino S, Haag-Wackernagel D, Geigenfeind I, Helmecke S, Dovc A, Prukner-Radovcic E, et al. 2009. Chlamydial infections in feral pigeons in Europe: review of data and focus on public health implications. Vet Microbiol 135: 54-67. doi: 10.1016/j.vetmic.2008.09.045

18. Martínez KP, Tinetti PS. 2007. Determinación de anticuerpos contra Chlamydophila psittaci por la prueba de ELISA en aves psitácidas tropicales mantenidas en cautiverio en el área metropolitana de San Salvador. Tesis de Baciller. El Salvador: Univ. de El Salvador. $139 \mathrm{p}$.

19. Monsalve $S$, Miranda $R$, Mattar $S$. 2011. Primera evidencia de circulación de Chlamydophila psittaci en Colombia: posible riesgo de salud pública. Rev Salud Pública 13: 314-326. doi: 10.1590/ S0124-00642011000200013

20. Morais J, Coelho AC, dos Anjos Pires M. 2013. Psittacosis. In: Insights from Veterinary Medicine. 129-162. doi: $10.5772 / 53720$

21. Olson S. 1995. Types and nomenclature of two Chilean parrots from the voyage of HMS Blonde (1825). B Brit Ornitol Ckub 115: 235-239.

22. Pianciola L, González G, Mazzeo M, Navello M, Quidel N, Bulgheroni MF. 2010. Desempeño de la prueba de inmunofluorescencia directa en el diagnóstico del virus Influenza A (H1N1). Rev Panam Salud Publ 27: 452-454.

23. Prukner-Radovcic E, Horvatek D, Gottstein Ž, Grozdanic IC, Mazija H. 2005. Epidemiological investigation of Chlamydophila psittaci in pigeons and free-living birds in Croatia. Vet Res Commun 29: 17-21.

24. Ramsay EC. 2003. The psittacosis outbreak of 1929-1930. J Avian Med Surg 17: 235-237. doi: 10.1647/10826742(2003)017[0235:TPOO]2.0.CO;2

25. Raso TDF, Júnior ÂB, Pinto AA. 2002. Evidence of Chlamydophila psittaci infection in captive Amazon parrots in Brazil. J Zoo Wildlife Med 33: 118-121. doi: 10.1638/1042-7260(2002)033[0118:EOCPII]2.0.CO;2

26. Reither N. 1999. The prevalence of clamydiosis in pets birds in Norway diagnosed with ImmunoComb ${ }^{\circledR}$ ELISA kit. $5^{\text {th }}$ European A.A.V. Conference. Pisa, Italy. 
27. Reyes A. 2011. Estimación de la población de mascotas no tradicionales en la ciudad de Talca y caracterización de sus dueños. Tesis de Médico Veterinario. Talca, Chile: Univ. Santo Tomás. 74 p.

28. Ryll M, Kummerfeld N, Petersen A, Neumann U, Bendheim U. 1994. Comparative investigations employing two different Chlamydia psittaciantibody-detection systems in Psittacides suspicious for psittacosis. In: Deutsch Veterinarmedizinische Gesellschaft. Munich, Germany [en alemán]

29. Ruppanner R, Behymer DE, DeLong WJ, Franti CE, Schulz T. 1984. Enzyme immunoassay of Chlamydia in birds. Avian Dis 28: 608-615.
30. Sachse K, Vretou E, Livingstone M, Borel N, Pospischil A, Longbottom D. 2009. Recent developments in the laboratory diagnosis of chlamydial infections. Vet Microbiol 135: 2-21. doi: 10.1016/j.vetmic.2008.09.040

31. Schettler E, Fickel J, Hotzel H, Sachse $K$, Streich WJ, Wittstatt U, Frölich $K$. 2003. Newcastle disease virus and Chlamydia psittaci in free-living raptors from eastern Germany. J Wildlife Dis 39: 57-63. doi: 10.7589/0090-3558-39.1.57

32. Talazadeh F, Mayahi M, Seifi M, Pourmehdi M. 2014. Evaluation of a commercial ELISA kit (IDEXX) to differentiate AI virus-infected poultry from AI-vaccinated poultry (DIVA). Rev Bras Cienc Avic 16: 73-77. doi: 10.1590/ $1516-635 \times 160273-78$ 